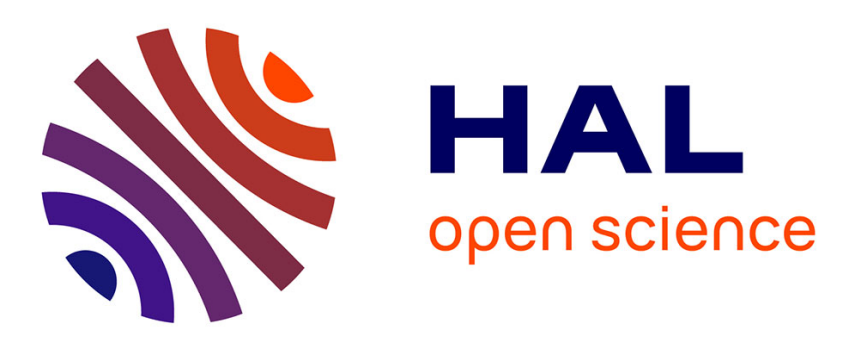

\title{
SOLVING A CROP PROBLEM BY AN OPTIMAL CONTROL METHOD
}

\author{
Maïtine Bergounioux, Hem Raj Joshi, Suzanne Sl Lenhart
}

\section{To cite this version:}

Maïtine Bergounioux, Hem Raj Joshi, Suzanne Sl Lenhart. SOLVING A CROP PROBLEM BY AN OPTIMAL CONTROL METHOD. Natural Resource Modeling, 2005, 18, number 3 pp 323-346. hal-00022001

\section{HAL Id: hal-00022001 https://hal.science/hal-00022001}

Submitted on 31 Mar 2006

HAL is a multi-disciplinary open access archive for the deposit and dissemination of scientific research documents, whether they are published or not. The documents may come from teaching and research institutions in France or abroad, or from public or private research centers.
L'archive ouverte pluridisciplinaire HAL, est destinée au dépôt et à la diffusion de documents scientifiques de niveau recherche, publiés ou non, émanant des établissements d'enseignement et de recherche français ou étrangers, des laboratoires publics ou privés. 


\title{
Solving a Crop Problem by an Optimal Control Method
}

\author{
Hem Raj Joshi* Suzanne Lenhart* Maïtine Bergounioux ${ }^{\dagger}$
}

(Draft)

Last Update : May $10^{\text {th }}, 2004$

\section{Résumé}

A system of ordinary differential equations coupled with a parabolic partial differential equation is studied in order to understand an interaction between two crops and a pathogen. Two different types of crops are planted in same field in some pattern so that the spread of pathogen can be controlled. The pathogen prefers to eat one crop. The other crop, which is not preferred by pathogen, is introduced to control the spread of pathogen in the farming land. The "optimal" initial planting pattern is sought to maximize plant yields while minimizing the variation in the planting pattern. The optimal pattern is characterized by a variation inequality involving the solutions of the optimality system. Numerical examples are given to illustrate the results.

Key Words : Crop Problem, Optimal Control, Pathogen Spread

AMS Classification : $\quad 35 \mathrm{~K} 55, \quad 49 \mathrm{~K} 20 \quad, 92 \mathrm{D} 25$

\footnotetext{
${ }^{*}$ University of Tennessee, Department of Mathematics, Knoxville, TN 37996-1300

${ }^{\dagger}$ Départment de Mathématiques, Université d'Orléans, Laboratoire MAPMO, UFR Sciences, BP 7659, 45067 Orléans, France.
} 


\section{Introduction}

We consider a system with two ordinary differential equations (ODEs) and one parabolic partial differential equation (PDE) modeling the planting of two different types of crops ( $u$ and $v$ ) in same field in some pattern so that the spread of pathogen $(w)$ can be controlled. The pathogen prefers to eat crop $u$. The crop $v$, which is not preferred by pathogen, is introduced to control the spread of pathogen in the farming land $(\Omega=(0,1))$.

The state system is the following :

$$
\begin{array}{ccc}
\frac{d u}{d t} & =r_{1} u\left(k_{1}-u\right)-k_{3} u w & \text { in } Q=\Omega \times(0, T) \\
\frac{d v}{d t} & =r_{2} v\left(k_{2}-v\right)-k_{4} v w & \\
w_{t}=d_{1} w_{x x}+\alpha_{1} k_{3} u w+\alpha_{2} k_{4} v w-\mu w &
\end{array}
$$

with initial and boundary conditions :

$$
\begin{aligned}
& u(x, 0)=u_{0}(x), \quad v(x, 0)=v_{0}(x)=a-u_{0}(x) \quad w(x, 0)=w_{0}(x) \quad \text { for } x \in \Omega \\
& \begin{array}{ll}
w(0, t)=0=w(1, t) & \text { on } \partial \Omega \times(0, T) .
\end{array}
\end{aligned}
$$

The coefficients and terms can be interpreted as :

$$
\begin{array}{ll}
u(x, t) & =\text { crop preferred by pathogen (first state variable) } \\
v(x, t) & =\text { crop not preferred by pathogen (second state variable) } . \\
w(x, t) & =\text { pathogen (third state variable). } \\
r_{1}, r_{2} & =\text { growth rates. } \\
d_{1} & =\text { diffusion coefficient. } \\
k_{1}, k_{2} & =\text { the carrying capacities. } \\
\alpha_{1}, \alpha_{2}, k_{3}, k_{4} & =\text { interaction coefficients. } \\
\mu & =\text { pathogen natural death rate. }
\end{array}
$$

The control set is

$$
U=\left\{u_{0}(x) \in H_{0}^{1}(0,1) \mid 0 \leq u_{0}(x) \leq a\right\}
$$

We seek to maximize the objective functional over $u_{0} \in U$ :

$$
J\left(u_{0}\right)=\int_{0}^{1}\left[\left(A_{1} u+A_{2} v\right)(x, T)-\frac{1}{2}\left(\left(u_{0}^{\prime}\right)^{2}+B_{1} u_{0}^{2}+B_{2}\left(a-u_{0}\right)^{2}\right)\right] d x .
$$

The positive constants $A_{1}$ and $A_{2}$ represent the relative importance of the terms $u$ and $v$ respectively and $B_{1}$ and $B_{2}$ are multipliers of the cost of implementing the control. Minimizing the $u_{0}^{\prime}$ term represents low variation in $u_{0}$. A planting pattern with high 
variation would be unrealistic to implement. The goal would be to maximize plant yields (subject to relative importance of the two crops) while minimizing the variation in the initial planting pattern.

Intercropping for weed and pest management has been considered in a variety of contexts $[5,8,9]$, but mostly in the setting of systems of ODEs differential equations or difference equations. Here we have a combination of a parabolic PDE for diffusion of the pathogen with ODEs for the crops. Due to inclusion of the $u^{\prime}$ variation term in the objective functional, our characterization of the optimal planting pattern is a variational inequality [7], instead of simply an algebraic expression in terms of the state and adjoint variables. Such a variational inequality is somewhat novel in control of PDE problems and requires an unusual numerical algorithm.

In section 2, we discuss the existence of an optimal control, i.e. the optimal planting pattern. The optimality system, which characterizes the optimal control, is derived in section 3 . The optimality system involves the state system and the adjoint system together with the characterization of the optimal control given by a variational inequality. Section 4 treats the uniqueness of the optimal control by obtaining the uniqueness of the solutions of the optimality system for $T$ sufficiently small. Finally in section 5 , we discuss our numerical algorithm and illustrate numerical examples for our problem.

\section{Existence of an Optimal Control}

The following assumptions are made throughout this paper.

1. $\alpha_{2} k_{4}<\mu$

2. $r_{1}, r_{2}, d_{1}, k_{3}, k_{4}, \alpha_{1}, \alpha_{2}, \mu$ are positive constants and $\alpha_{1} k_{3}>\alpha_{2} k_{4}$.

3. $0<a \leq 1$.

Assumption 1 means that the $u \equiv 0$ would cause the pathogen to decay when it only eats the second crop. Assumption 2 means that the consumption of crop $u$ contributes more to the growth of the pathogen than would comsumption of crop $v$.

The underlying solution space for system $(1.1)$ is $V=\left(L^{2}(Q)\right)^{2} \times L^{2}\left(0, T ; H_{0}^{1}(\Omega)\right)$.

Definition 2.1 We say a triple of functions $u, v, w \in V$, with $w_{t} \in L^{2}\left(0, T ; H^{-1}(\Omega)\right)$, is a weak solution of (1.1) with given boundary and initial conditions provided

$$
\begin{gathered}
u(x, t)=u_{0}(x)+\int_{0}^{t}\left[r_{1} u\left(k_{1}-u\right)-k_{3} u w\right](x, s) d s \\
v(x, t)=v_{0}(x)+\int_{0}^{t}\left[r_{2} v\left(k_{2}-v\right)-k_{4} v w\right](x, s) d s \\
\int_{0}^{T}<w_{t}, \phi>d t+d_{1} \int_{Q} \nabla u \nabla \phi d x d t
\end{gathered}
$$




$$
=\int_{Q}\left(\alpha_{1} k_{3} u w+\alpha_{2} k_{4} v w-\mu w\right) \phi d x d t
$$

for all $\phi \in L^{2}\left(0, T ; H_{0}^{1}(\Omega)\right)$, where the $<,>$ inner product is the duality between $H^{-1}(\Omega)$ and $H_{0}^{1}(\Omega)$, and

$$
u(x, 0)=u_{0}(x), \quad v(x, 0)=v_{0}(x) \quad w(x, 0)=w_{0}(x), \quad w(0, t)=0=w(1, t)
$$

Remark : Since $u, v \in C\left(0, T ; L^{2}(\Omega)\right)$ from Evans [1], the initial conditions (2.1) make sense.

We will prove an existence and uniqueness result for the state system (1.1). This result will be established in Theorem 2.1.

Theorem 2.1 Given $u_{0} \in U$, there exists a unique solution $(u, v, w)$ in $V$ solving (1.1) and (2.1).

Proof : We consider the following problems

$$
\begin{array}{cc}
\frac{d U}{d t}=r_{1} k_{1} U & \\
U(x, 0)=u_{0}(x) & \text { for } x \in \Omega \\
\frac{d V}{d t}=r_{2} k_{2} V & \\
V(x, 0)=v_{0}(x) & \text { for } x \in \Omega \\
W_{t}=d_{1} W_{x x}+\alpha_{1} k_{3} U W+\alpha_{2} k_{4} V W-\mu W & \\
W(x, 0)=w_{0}(x) & \text { for } x \in \Omega \\
W(0, t)=0=W(1, t) & \text { on } \partial \Omega \times(0, T) .
\end{array}
$$

The functions $U, V, W$ are supersolutions of system equations in (1.1), which are $L^{\infty}$ bounded in $Q$. To obtain the existence, we will construct three sequences by means of iteration, using the above supersolutions.

Define : $u^{1}=U, v^{1}=V, w^{2}=W, u^{0}=0, v^{0}=0, w^{1}=0$, where the superscripts denote the iteration step. For $i=2,3, \cdots$, we define $u^{i}, v^{i}$ and $w^{i+1}$ as the solution of the following problems respectively :

$$
\begin{array}{cl}
u_{t}^{i}+R u^{i}=f\left(u^{i-2}, w^{i}\right) & \text { in } Q \\
u^{i}(x, 0)=u_{0}(x) & \text { for } x \in \Omega \\
v_{t}^{i}+R v^{i}=g\left(v^{i-2}, w^{i}\right) & \text { in } Q \\
v^{i}(x, 0)=v_{0}(x) & \text { for } x \in \Omega \\
w_{t}^{i}-d_{1} w_{x x}^{i}+R w^{i}=h\left(u^{i-1}, v^{i-1}, w^{i-2}\right) & \text { in } Q \\
w^{i}(x, 0)=w_{0}(x) & \text { for } x \in \Omega \\
w^{i}(0, t)=0=w^{i}(1, t) & \text { on } \partial \Omega \times(0, T) .
\end{array}
$$




$$
\begin{array}{ll}
\text { where } & f\left(u^{i-2}, w^{i}\right)=R u^{i-2}+r_{1} u^{i-2}\left(k_{1}-u^{i-2}\right)-k_{3} u^{i-2} w^{i} \\
& g\left(v^{i-2}, w^{i}\right)=R v^{i-2}+r_{2} v^{i-2}\left(k_{2}-v^{i-2}\right)-k_{4} v^{i-2} w^{i} \\
& h\left(u^{i-1}, v^{i-1}, w^{i-2}\right)=R w^{i-2}+\alpha_{1} k_{3} u^{i-1} w^{i-2}+\alpha_{2} k_{4} v^{i-1} w^{i-2}-\mu w^{i-2} .
\end{array}
$$

$R$ is a constant satisfying $\sup _{Q}\left(\left(k_{3}+k_{4}+\mu\right) W+2 r_{1} U+2 r_{2} V\right)$.

Since $(2.2),(2.2)$ and $(2.2)$ are linear problems, the solutions $u^{i}, v^{i}, w^{i}$, for $i=1,2, \cdots$, exist.

\section{Claim 1 :}

For $i=1,2, \cdots, 0 \leq u^{i} \leq U, 0 \leq v^{i} \leq V, 0 \leq w^{i} \leq W$ in $Q$.

Claim 1 can be proved by induction using the maximum principle.

Note that for $u^{i} \geq 0, v^{i} \geq 0, w^{i} \geq 0, i=1,2, \cdots$,

$f$ is increasing in $u^{i-2}$, and decreasing in $w^{i}$.

$g$ is increasing in $v^{i-2}$, and decreasing in $w^{i}$.

$h$ is increasing in $u^{i-1}, v^{i-1}$ and decreasing in $w^{i-2}$.

\section{Claim 2 :}

There exists $\bar{u}, \bar{v}, \bar{w}, u, v, w$ in $V$ such that the following monotone pointwise convergence holds.

$$
\begin{array}{lll}
u^{2 i} \nearrow \bar{u}, & u^{2 i+1} \searrow u & \text { in } Q \\
v^{2 i} \nearrow \bar{v}, & v^{2 i+1} \searrow v & \text { in } Q \\
w^{2 i} \searrow \bar{w}, & w^{2 i+1} \nearrow w & \text { in } Q .
\end{array}
$$

\section{Proof of Claim 2 :}

To prove the convergence we use the induction method. Since $w^{1}=0$, we have

$$
\left(w^{5}-w^{3}\right)_{t}-d_{1}\left(w^{5}-w^{3}\right)_{x x}+R\left(w^{5}-w^{3}\right)=w^{3}\left(R+\alpha_{1} k_{3} u^{4}+\alpha_{2} k_{4} v^{4}-\mu\right) \geq 0
$$

and thus $0 \leq w^{1} \leq w^{3} \leq w^{5}$. Similarly we have $u^{3} \leq u^{1}, v^{3} \leq v^{1}, w^{2} \geq w^{4}, u^{0} \leq u^{2}$ and $v^{0} \leq v^{2}$. Also $u^{2} \leq u^{3}, v^{2} \leq v^{3}$ and $w^{4} \geq w^{3}$ since

$$
\begin{gathered}
\left(u^{2}-u^{3}\right)_{t}+R\left(u^{2}-u^{3}\right)=-u^{1}\left(R+r_{1}\left(k_{1}-u^{1}\right)-k_{3} w^{3}\right) \leq 0 \\
\left(v^{2}-v^{3}\right)_{t}+R\left(v^{2}-v^{3}\right)=-v^{1}\left(R+r_{2}\left(k_{2}-v^{1}\right)-k_{4} w^{3}\right) \leq 0 \\
\left(w^{4}-w^{3}\right)_{t}-d_{1}\left(w^{4}-w^{3}\right)_{x x}+R\left(w^{4}-w^{3}\right)=w^{2}\left(R+\alpha_{1} k_{3} u^{3}+\alpha_{2} k_{4} v^{3}-\mu\right) \geq 0 .
\end{gathered}
$$

Fix $i$, assume that for all $j \leq i-1$ such that

$$
\begin{array}{ll}
u^{2 j} \nearrow & u^{2 j+1} \searrow \\
v^{2 j} \nearrow & v^{2 j+1} \searrow \\
w^{2 j} \searrow & w^{2 j+1} \nearrow .
\end{array}
$$


Now we compare $w^{2 i+1}$ and $w^{2 i}$. From (2.2), we have

$$
\begin{gathered}
\left(w^{2 i+2}-w^{2 i}\right)_{t}-d_{1}\left(w^{2 i+2}-w^{2 i}\right)_{x x}+R\left(w^{2 i+2}-w^{2 i}\right) \\
=h\left(u^{2 i+1}, v^{2 i+1}, w^{2 i}\right)-h\left(u^{2 i-1}, v^{2 i-1}, w^{2 i-2}\right) \leq 0 .
\end{gathered}
$$

Then $w^{2 i+2} \leq w^{2 i}$. Other cases can be proved using similar arguments. Hence by boundedness of $u^{i}, v^{i}, w^{i}$ and monotone properties of $f, g, h$, we get the pointwise convergence.

\section{Claim 3 :}

The subsequences of $\left\{u^{i}\right\},\left\{v^{i}\right\},\left\{w^{i}\right\}$ satisfy :

$u^{2 j} \rightarrow \bar{u}, \quad u^{2 j+1} \rightarrow u, \quad v^{2 j} \rightarrow \bar{v}, \quad v^{2 j+1} \rightarrow v, \quad w^{2 j} \rightarrow \bar{w}, \quad w^{2 j+1} \rightarrow w \quad$ weakly in $V$.

\section{Proof of Claim 3 :}

Since RHS of (2.2), (2.2) and (2.2) are bounded in $L^{\infty}(Q)$, then the sequences

$$
\left\{\left(u^{2 i}, v^{2 i}, w^{2 i}\right)\right\},\left\{\left(u^{2 i+1}, v^{2 i+1}, w^{2 i+1}\right)\right\}
$$

are uniformly bounded in $V$.

Hence using the weak compactness of the sequences in $V$, we get $u^{2 j} \rightarrow \bar{u}, \quad u^{2 j+1} \rightarrow u, \quad v^{2 j} \rightarrow \bar{v}, \quad v^{2 j+1} \rightarrow v, \quad w^{2 j} \rightarrow \bar{w}, \quad w^{2 j+1} \rightarrow w \quad$ weakly in $V$.

Since we have pointwise convergence on each sequence by claim 2 , this weak convergence is also on the whole (even or odd) sequence (not just on a subsequence).

\section{Claim 4}

The subsequences of $\left\{u^{i}\right\},\left\{v^{i}\right\},\left\{w^{i}\right\}$ satisfy

$$
u^{2 j} \rightarrow \bar{u}, u^{2 j+1} \rightarrow u, v^{2 j} \rightarrow \bar{v}, v^{2 j+1} \rightarrow v, w^{2 j} \rightarrow \bar{w}, w^{2 j+1} \rightarrow w \text { strongly in } L^{2}(Q) .
$$

\section{Proof of Claim 4 :}

Using the $V$ boundedness on $u^{2 i}, u^{2 i+1}, v^{2 i}, v^{2 i+1}, w^{2 i}, w^{2 i+1}$ and the system in (2.2)(2.2), we obtain that

$$
\left\|w_{t}^{2 i}\right\|,\left\|w_{t}^{2 i+1}\right\| \text { are bounded in } L^{2}\left(0, T ; H^{-1}(\Omega)\right) .
$$

Hence, using weak compactness again, we have

$$
w_{t}^{2 i} \rightarrow \bar{w}_{t}, \quad w_{t}^{2 i+1} \rightarrow w_{t} \quad \text { weakly in } L^{2}\left(0, T ; H^{-1}(\Omega)\right) .
$$

Using a compactness result by Simon [10] for $w$ and, we have

$$
w^{2 i} \rightarrow \bar{w}, \quad w^{2 i+1} \rightarrow w \quad \text { strongly in } L^{2}(Q) .
$$


Since equations (2.2), (2.2) are ODEs in time with parameters $x$ and bounded RHS, the sequence is uniformly Lipschitz in $t$ for each $x$, we can pass to limit in $u, v$ by using weak solution definition

$u^{2 i} \rightarrow \bar{u}, \quad u^{2 i+1} \rightarrow u, \quad v^{2 i} \rightarrow \bar{v}, \quad v^{2 i+1} \rightarrow v \quad$ pointwise uniformly in $t$, for each $x$

Now we prove the uniqueness, i. e., $u=\bar{u}, v=\bar{v}$ and $w=\bar{w}$. Passing to the limit in the $u^{2 i}, u^{2 i+1}, v^{2 i}, v^{2 i+1}, w^{2 i}$, and $w^{2 i+1} \mathrm{PDE}$ 's, we obtain :

$$
\begin{aligned}
\bar{u}_{t} & =r_{1} k_{1} \bar{u}-r_{1} \bar{u}^{2}-k_{3} \overline{u w} & & \text { in } Q \\
\bar{u}(x, 0) & =u_{0}(x) & & \text { for } x \in \Omega \\
u_{t} & =r_{1} k_{1} u-r_{1} u^{2}-k_{3} u w & & \text { in } Q \\
u(x, 0) & =u_{0}(x) & & \text { for } x \in \Omega \\
\bar{v}_{t} & =r_{2} k_{2} \bar{v}-r_{2} \bar{v}^{2}-k_{4} \overline{v w} & & \text { in } Q \\
\bar{v}(x, 0) & =v_{0}(x) & & \text { for } x \in \Omega \\
v_{t} & =r_{2} k_{2} v-r_{2} v^{2}-k_{4} v w & & \text { in } Q \\
v(x, 0) & =v_{0}(x) & & \text { for } x \in \Omega \\
\bar{w}_{t}-d_{1} \bar{w}_{x x} & =\alpha_{1} k_{3} u \bar{w}+\alpha_{2} k_{3} v \bar{w}-\mu \bar{w} & & \text { in } Q \\
\bar{w}(0, t) & =0=\bar{w}(1, t) & & \text { on } \partial \Omega \times(0, T) \\
\bar{w}(x, 0) & =w_{0}(x) & & \text { for } x \in \Omega \\
w_{t}-d_{1} w_{x x} & =\alpha_{1} k_{3} \bar{u} w+\alpha_{2} k_{3} \bar{v} w-\mu w & & \text { in } Q \\
w(0, t) & =0=w(1, t) & & \text { on } \partial \Omega \times(0, T) \\
w(x, 0) & =w_{0}(x) & & \text { for } x \in \Omega
\end{aligned}
$$

Let $u=e^{\lambda t} f, \bar{u}=e^{\lambda t} \bar{f}, v=e^{\lambda t} g, \bar{v}=e^{\lambda t} \bar{g}, w=e^{\lambda t} h$ and $\bar{w}=e^{\lambda t} \bar{h}$, where $\lambda>0$ is to be chosen. To illustrate the transformed system, we write equation (2.2) :

$$
f_{t}+\lambda f=r_{1} k_{1} f-r_{1} e^{\lambda t} f^{2}-k_{3} e^{\lambda t} f h \quad \text { in } Q
$$

We consider the weak formulation of the $f-\bar{f}, g-\bar{g}$ and $h-\bar{h}$ problems, and after adding both weak formulations, we obtain on $Q_{1}=\Omega \times\left(0, t_{1}\right)$ :

$$
\begin{gathered}
\int_{Q_{1}}\left\{(f-\bar{f})_{t}(f-\bar{f})+\lambda(f-\bar{f})^{2}+(g-\bar{g})_{t}(g-\bar{g})+\lambda(g-\bar{g})^{2}\right\} d x d t \\
+\int_{Q_{1}}\left\{(h-\bar{h})_{t}(h-\bar{h})+d_{1}|\nabla(h-\bar{h})|^{2}+\lambda(h-\bar{h})^{2}\right\} d x d t \\
=\int_{Q_{1}}\left\{r_{1} k_{1}(f-\bar{f})^{2}-r_{1} e^{\lambda t}\left(f^{2}-\bar{f}^{2}\right)(f-\bar{f})-k_{3} e^{\lambda t}(f-\bar{f})(f h-\bar{f} \bar{h})\right\} d x d t \\
+\int_{Q_{1}}\left\{r_{2} k_{2}(g-\bar{g})^{2}-r_{2} e^{\lambda t}\left(g^{2}-\bar{g}^{2}\right)(g-\bar{g})-k_{4} e^{\lambda t}(g-\bar{g})(g h-\bar{g} \bar{h})\right\} d x d t \\
+\int_{Q_{1}}\left\{\alpha_{1} k_{3}(\bar{f} h-f \bar{h})(h-\bar{h})+\alpha_{2} k_{4}(\bar{g} h-g \bar{h})(h-\bar{h})-\mu(h-\bar{h})^{2}\right\} d x d t .
\end{gathered}
$$


We obtain

$$
\begin{gathered}
\frac{1}{2} \int_{\Omega}\left\{[f-\bar{f}]^{2}(x, T)+[g-\bar{g}]^{2}(x, T)+[h-\bar{h}]^{2}(x, T)\right\} d x+\int_{Q_{1}}\left\{\left[d_{1}(|\nabla(h-\bar{h})|)^{2}\right] d x d t\right. \\
+\left(\lambda-C_{1}-C_{2} e^{\lambda t_{1}}\right) \int_{Q_{1}}\left[(f-\bar{f})^{2}+(g-\bar{g})^{2}+(h-\bar{h})^{2}\right] d x d t \leq 0
\end{gathered}
$$

where $C_{1}, C_{2}$ depend on the coefficients and the solution bounds.

If we choose $\lambda>C_{1}+C_{2}$ and $t_{1}$ such that $t_{1}<\frac{1}{\lambda} \ln \left(\frac{\lambda-C_{1}}{C_{2}}\right)$, then inequality (2.9) holds if and only if

$$
f=\bar{f}, \quad g=\bar{g}, \quad w=\bar{w} \quad \text { a.e. in } Q .
$$

Similarly the proof can be completed for time intervals $\left(\left[t_{1}, 2 t_{1}\right],\left[2 t_{1}, 3 t_{1}\right] \ldots\right)$. Therefore, $u=\bar{u}, v=\bar{v}$ and $v=\bar{v}$ a.e. in $Q$, and $u, v, w$ solve the state system (1.1). Hence the solution to the state system (1.1) exists and the uniqueness of $u, v, w$ as solutions of (1.1) follows similarly as in the above argument.

Theorem 2.2 There exists an optimal control in $U$ that maximizes the functional $J\left(u_{0}\right)$. Proof $: \sup \left\{J\left(u_{0}\right) \mid u_{0} \in U\right\}<\infty$ since the state variables and controls are uniformly bounded. Thus there exists a maximizing sequence $u_{0} \in U$ such that

$$
\lim _{n \rightarrow \infty} J\left(u_{0}^{n}\right)=\sup \left\{J\left(u_{0}\right) \mid u_{0} \in U\right\} .
$$

By the existence and uniqueness of solutions to the state system (1.1), we define

$$
u^{n}=u\left(u_{0}^{n}\right), \quad v^{n}=v\left(u_{0}^{n}\right), \quad w^{n}=w\left(u_{0}^{n}\right) \quad \text { for each } n .
$$

On a subsequence, as $n \rightarrow \infty, u_{0}^{n} \rightarrow u_{0}^{*}$ in $L^{2}(Q)$ and $\left(u_{0}^{n}\right)^{\prime} \rightarrow\left(u_{0}^{*}\right)^{\prime}$ weakly in $L^{2}(Q)$ Passing to the limit in the $u^{n}, v^{n}, w^{n}$ system and using the convergences as in Theorem 2.1 , we have that $(u, v, w)$ is weak solution of (1.1) associated with $u_{0}^{*}$. Since the payoff functional is upper semi-continuous with respect to the weak convergence, we have

$$
J\left(u_{0}^{*}\right) \leq \sup \left\{J\left(u_{0}^{n}\right) \mid u_{0}^{n} \in U\right\} .
$$

Therefore $u_{0}^{*}$ is an optimal control that maximizes the payoff functional.

\section{Derivation of the Optimality System}

We now derive the optimality system which consists of the state system coupled with the adjoint system. In order to obtain the necessary conditions for the optimality system 
we differentiate the objective functional with respect to the control. As our objective functional also depends on state variables, we differentiate the state variables with respect to the control $u_{0}$

Theorem 3.1 The mapping $u_{0} \in U \rightarrow(u, v, w) \in V$ is differentiable in the following sense :

$$
\begin{array}{ccc}
\frac{u\left(u_{0}+\epsilon l\right)-u\left(u_{0}\right)}{\epsilon} & \rightarrow \psi_{1} \quad \text { weakly in } L^{2}(Q) \\
\frac{v\left(u_{0}+\epsilon l\right)-v\left(u_{0}\right)}{\epsilon} & \rightarrow \psi_{2} \quad \text { weakly in } L^{2}(Q) \\
\frac{w\left(u_{0}+\epsilon l\right)-w\left(u_{0}\right)}{\epsilon} & \rightarrow \psi_{3} \quad \text { weakly in } L^{2}\left(0, T ; H_{0}^{1}(\Omega)\right)
\end{array}
$$

as $\epsilon \rightarrow 0$ for any $u_{0} \in U$ and $l \in L^{\infty}(Q)$ s.t. $\left(u_{o}+\epsilon l\right) \in U$ for $\epsilon$ small. Also $\psi_{1}, \psi_{2}, \psi_{3}$ (depending on $u, v, w, u_{0}, l$ ) satisfy the following system :

$$
\begin{aligned}
& \left(\psi_{1}\right)_{t} \quad=r_{1}\left(k_{1}-2 u\right) \psi_{1}-k_{3}\left(u \psi_{3}+w \psi_{1}\right) \quad \text { in } Q \\
& \left(\psi_{2}\right)_{t} \quad=r_{2}\left(k_{2}-2 v\right) \psi_{2}-k_{4}\left(v \psi_{3}+w \psi_{2}\right) \quad \text { in } Q \\
& \left(\psi_{3}\right)_{t}=d_{1}\left(\psi_{3}\right)_{x x}+\alpha_{1} k_{3}\left(u \psi_{3}+w \psi_{1}\right)+\alpha_{2} k_{4}\left(v \psi_{3}+w \psi_{2}\right)-\mu \psi_{3} \quad \text { in } Q \\
& \psi_{1}(x, 0) \quad=l, \quad \psi_{2}(x, 0)=-l, \quad \psi_{3}(x, 0)=0 \quad \text { for } x \in \Omega \\
& \psi_{3}(0, t) \quad=0=\psi_{3}(1, t) \quad \text { on } \quad \partial \Omega \times(0, T)
\end{aligned}
$$

Proof : Define $u^{\epsilon}=u\left(u_{0}+\epsilon l\right), v^{\epsilon}=v\left(u_{0}+\epsilon l\right), u=u\left(u_{0}\right), v=v\left(u_{0}\right)$ and $w=w\left(u_{0}\right)$. We do a change of variables : $u^{\epsilon}=e^{\lambda t} f^{\epsilon}, u=e^{\lambda t} f, v^{\epsilon}=e^{\lambda t} g^{\epsilon}, v=e^{\lambda t} g, w^{\epsilon}=e^{\lambda t} h^{\epsilon}, w=e^{\lambda t} h$, where $\lambda>0$ is to be chosen below.

On the set $Q_{1}=\Omega \times\left(0, t_{1}\right)$ for $0<t_{1} \leq T$, we illustrate the " $h$ " equation :

$$
\begin{gathered}
\int_{Q_{1}}\left[\left(\frac{h^{\epsilon}-h}{\epsilon}\right)\left(\frac{h^{\epsilon}-h}{\epsilon}\right)_{t}+\lambda\left(\frac{h^{\epsilon}-h}{\epsilon}\right)^{2}+d_{1}\left|\left(\frac{h^{\epsilon}-h}{\epsilon}\right)_{x}\right|^{2}\right] d x d t \\
=\int_{Q_{1}}\left[\alpha_{1} k_{3} e^{\lambda t}\left(f^{\epsilon}\left(\frac{h^{\epsilon}-h}{\epsilon}\right)^{2}+h\left(\frac{f^{\epsilon}-f}{\epsilon}\right)\left(\frac{h^{\epsilon}-h}{\epsilon}\right)\right)\right. \\
\left.+\alpha_{2} k_{4} e^{\lambda t}\left(g^{\epsilon}\left(\frac{h^{\epsilon}-h}{\epsilon}\right)^{2}+h\left(\frac{g^{\epsilon}-g}{\epsilon}\right)\left(\frac{h^{\epsilon}-h}{\epsilon}\right)\right)-\mu\left(\frac{h^{\epsilon}-h}{\epsilon}\right)^{2}\right] d x d t
\end{gathered}
$$

Continuing to estimate using $L^{\infty}$ bounds on the coefficients and $f, g, h, f^{\epsilon}, g^{\epsilon}$ and $h^{\epsilon}$, we have

$$
\begin{gathered}
\frac{1}{2} \int_{\Omega \times\left\{t_{1}\right\}}\left[\left(\frac{f^{\epsilon}-f}{\epsilon}\right)^{2}+\left(\frac{g^{\epsilon}-g}{\epsilon}\right)^{2}+\left(\frac{h^{\epsilon}-h}{\epsilon}\right)^{2}\right] d x+\int_{Q_{1}} d_{1}\left|\left(\frac{h^{\epsilon}-h}{\epsilon}\right)_{x}\right|^{2} d x d t \\
+\left(\lambda-\left(C_{1}+C_{2} e^{\lambda t_{1}}\right)\right) \int_{Q_{1}}\left[\left(\frac{f^{\epsilon}-f}{\epsilon}\right)^{2}+\left(\frac{g^{\epsilon}-g}{\epsilon}\right)^{2}+\left(\frac{h^{\epsilon}-h}{\epsilon}\right)^{2}\right] d x d t \\
\leq C \int_{\Omega} l^{2} d x .
\end{gathered}
$$


For $\lambda>C_{1}+C_{2}$ and $t_{1}$ small such that $t_{1}<\frac{1}{\lambda} \ln \frac{\lambda-C_{1}}{C_{2}}$, we conclude

$$
\left\|\frac{f^{\epsilon}-f}{\epsilon}\right\|_{L^{2}\left(Q_{1}\right)}^{2}+\left\|\frac{g^{\epsilon}-g}{\epsilon}\right\|_{L^{2}\left(Q_{1}\right)}^{2}+\left\|\frac{h^{\epsilon}-h}{\epsilon}\right\|_{L^{2}\left(0, t_{1} ; H_{0}^{1}(\Omega)\right.}^{2} \leq C \int_{\Omega} l^{2} d x .
$$

Similarly this estimate can be carried out on intervals $\left[t_{1}, 2 t_{1}\right],\left[2 t_{1}, 3 t_{1}\right], \cdots$ and the estimate finally holds on $[0, T]$. This estimate justifies the convergence of $f, g$ and $h$ quotients, and hence

$$
\begin{array}{ll}
\frac{u^{\epsilon}-u}{\epsilon} & \rightarrow \psi_{1} \quad \text { weakly in } L^{2}(Q) \\
\frac{v^{\epsilon}-v}{\epsilon} & \rightarrow \psi_{2} \quad \text { weakly in } L^{2}(Q) \\
\frac{w^{\epsilon}-w}{\epsilon} & \rightarrow \psi_{3} \quad \text { weakly in } L^{2}\left(0, T ; H_{0}^{1}(\Omega)\right)
\end{array}
$$

Similarly we obtain

$$
\left(\frac{w^{\epsilon}-w}{\epsilon}\right)_{t} \rightarrow\left(\psi_{3}\right)_{t} \quad \text { weakly in } L^{2}\left(0, T ; H^{-1}(\Omega)\right)
$$

and

$$
\frac{w^{\epsilon}-w}{\epsilon} \rightarrow \psi_{3}, \quad \text { strongly in } L^{2}(Q)
$$

$\frac{u^{\epsilon}-u}{\epsilon}, \quad \frac{v^{\epsilon}-v}{\epsilon} \quad$ are uniformly Lipschitz in $t$ for each $x$.

These convergences also give $u^{\epsilon} \rightarrow u, v^{\epsilon} \rightarrow v, w^{\epsilon} \rightarrow w$ strongly in $L^{2}(Q)$.

To see the system satisfied by $\psi_{1}, \psi_{2}, \psi_{3}$, consider terms from the system satisfied by $\frac{u^{\epsilon}-u}{\epsilon}, \frac{v^{\epsilon}-v}{\epsilon}$ and $\frac{w^{\epsilon}-w}{\epsilon}$; for example

$$
\begin{gathered}
\frac{1}{\epsilon} r_{1}\left(\left(u^{\epsilon}\right)^{2}-u^{2}\right)=r_{1} \frac{1}{\epsilon}\left(u^{\epsilon}-u\right)\left(u^{\epsilon}+u\right) \rightarrow 2 r_{1} u \psi_{1} \quad \text { as } \epsilon \rightarrow 0, \\
\frac{1}{\epsilon} k_{3}\left(u^{\epsilon} w^{\epsilon}-u w\right)=k_{3} \frac{1}{\epsilon}\left(u^{\epsilon}\left(w^{\epsilon}-w\right)+w\left(u^{\epsilon}-u\right)\right) \rightarrow k_{3}\left(u \psi_{3}+w \psi_{1}\right),
\end{gathered}
$$

since $u^{\epsilon} \rightarrow u, w^{\epsilon} \rightarrow w$ as $\epsilon \rightarrow 0$.

The above estimates justify passing the limit in the system satisfied by $\frac{u^{\epsilon}-u}{\epsilon}, \frac{v^{\epsilon}-v}{\epsilon}$ and $\frac{w^{\epsilon}-w}{\epsilon}$, and we conclude that $\psi_{1}, \psi_{2}, \psi_{3}$ solves (3.1).

To derive the optimality system and to characterize the pairs of optimal controls, we need adjoints and adjoints of the operators associated with $\psi_{1}, \psi_{2}, \psi_{3}$ system as

$$
\mathcal{L}\left(\begin{array}{l}
\psi_{1} \\
\psi_{2} \\
\psi_{3}
\end{array}\right)=\left(\begin{array}{l}
0 \\
0 \\
0
\end{array}\right)
$$


where

$$
\begin{gathered}
\mathcal{L}\left(\begin{array}{l}
\psi_{1} \\
\psi_{2} \\
\psi_{3}
\end{array}\right)=\left(\begin{array}{c}
\mathcal{L}_{1} \psi_{1} \\
\mathcal{L}_{2} \psi_{2} \\
\mathcal{L}_{3} \psi_{3}
\end{array}\right)-M\left(\begin{array}{l}
\psi_{1} \\
\psi_{2} \\
\psi_{3}
\end{array}\right) \\
\left(\begin{array}{c}
\mathcal{L}_{1} \psi_{1} \\
\mathcal{L}_{2} \psi_{2} \\
\mathcal{L}_{3} \psi_{3}
\end{array}\right)=\left(\begin{array}{c}
\left(\psi_{1}\right)_{t} \\
\left(\psi_{2}\right)_{t} \\
\left(\psi_{3}\right)_{t}-d_{1}\left(\psi_{3}\right)_{x x}
\end{array}\right) \\
M=\left(\begin{array}{lll}
r_{1}\left(k_{1}-2 u\right)-k_{3} w & 0 \\
0 & r_{2}\left(k_{2}-2 v\right)-k_{4} w & -k_{4} v \\
\alpha_{1} k_{3} w & \alpha_{2} k_{4} w & \alpha_{1} k_{3} u+\alpha_{2} k_{4} v-\mu
\end{array}\right) .
\end{gathered}
$$

We define the adjoint PDE system as $\mathcal{L}^{*}\left(\begin{array}{l}p \\ q \\ r\end{array}\right)=\left(\begin{array}{l}0 \\ 0 \\ 0\end{array}\right)$

where

$$
\mathcal{L}^{*}\left(\begin{array}{c}
p \\
q \\
r
\end{array}\right)=\left(\begin{array}{c}
\mathcal{L}_{1}^{*} p \\
\mathcal{L}_{2}^{*} q \\
\mathcal{L}_{3}^{*} r
\end{array}\right)-M^{\tau}\left(\begin{array}{c}
p \\
q \\
r
\end{array}\right)
$$

with

$$
\left(\begin{array}{l}
\mathcal{L}_{1}^{*} p \\
\mathcal{L}_{2}^{*} q \\
\mathcal{L}_{3}^{*} r
\end{array}\right)=\left(\begin{array}{l}
-p_{t} \\
-q_{t} \\
-r_{t}-d_{1} r_{x x}
\end{array}\right)
$$

and $M^{\tau}$ is the transpose of matrix $M$. Note that $A_{1}, A_{2}$ from the objective functional will occur as final time values for $p, q$.

To clarify the characterization of our optimal control, we make the following definition involving a variational inequality with upper and lower obstacles [3] :

Definition $3.1 u_{0} \in U$ is a weak solution of the following bilateral variational inequality

$$
\min \left\{\max \left(-\left(p(x, 0)-q(x, 0)+\left(u_{0}\right)_{x x}-B_{1} u_{0}+B_{2}\left(a-u_{0}\right)\right), u_{0}-a\right), u_{0}-0\right\}=0
$$

if for all $v_{0} \in U$,

$$
\int_{\Omega}\left[\left(u_{0}\right)_{x}\left(v_{0}-u_{0}\right)_{x}+\left\{q(x, 0)-p(x, 0)+B_{1} u_{0}-B_{2}\left(a-u_{0}\right)\right\}\left(v_{0}-u_{0}\right)\right] d x \geq 0 .
$$

\section{Theorem 3.2 Existence of Weak Solution}

Given an optimal control $u_{0}$ and corresponding solution $(u, v, w)=\left(u\left(u_{0}\right), v\left(u_{0}\right), w\left(u_{0}\right)\right)$ 
there exists a weak solution $(p, q, r) \in\left(L^{2}(Q)\right)^{2} \times L^{2}\left(0, T ; H_{0}^{1}(\Omega)\right)$ satisfying the adjoint system :

$$
\begin{gathered}
\mathcal{L}_{1}^{*} p=\left(r_{1}\left(k_{1}-2 u\right)-k_{3} w\right) p+\alpha_{1} k_{3} w r \\
\mathcal{L}_{2}^{*} q=\left(r_{2}\left(k_{2}-2 v\right)-k_{4} w\right) q+\alpha_{2} k_{4} w r \\
\mathcal{L}_{3}^{*} r=-k_{3} u p-k_{4} v q+\left(\alpha_{1} k_{3} u+\alpha_{2} k_{4} v-\mu\right) r
\end{gathered}
$$

and transversality conditions

$$
\begin{gathered}
p(x, T)=A_{1}, \quad q(x, T)=A_{2}, \quad r(x, T)=0 \quad \text { where } x \in \Omega \\
r(0, t)=0=r(1, t) \quad \text { where } t \in[0, T] .
\end{gathered}
$$

And furthermore $u_{0}(x)$ must satisfy the following variational inequality in the weak sense.

$$
\min \left\{\max \left(-\left(p(x, 0)-q(x, 0)+\left(u_{0}\right)_{x x}-B_{1} u_{0}+B_{2}\left(a-u_{0}\right)\right), u_{0}-a\right), u_{0}-0\right\}=0
$$

Proof : Let $u_{0}(x)$ be an optimal control (which exists by Theorem 2.2) and $(u, v, w)$ be its corresponding state solution. Let $\left(u_{0}(x)+\epsilon l\right) \in U$ for $\epsilon>0$, and $u^{\epsilon}, v^{\epsilon}, w^{\epsilon}$ be the corresponding weak solution of state system (1.1). Since the adjoint equations are linear, there exists a weak solution $p, q, r$ satisfying $(3.2-3.2)$. We compute the directional derivative of the objective functional $J\left(u_{0}\right)$ with respect to $u_{0}$ in the direction $l$ at $u_{0}$. Since $J\left(u_{0}\right)$ is the maximum value, we have

0

$$
\begin{gathered}
\geq \lim _{\epsilon \rightarrow 0^{+}} \frac{J\left(u_{0}(x)+\epsilon l\right)-J\left(u_{0}\right)}{\epsilon} \\
=\lim _{\epsilon \rightarrow 0^{+}}\left\{\int_{0}^{1}\left[A_{1}\left(\frac{u^{\epsilon}-u}{\epsilon}\right)+A_{2}\left(\frac{v^{\epsilon}-v}{\epsilon}\right)\right](x, T) d x-\frac{1}{2} \int_{0}^{1} \frac{\left(\left(u_{0}+\epsilon l\right)_{t}\right)^{2}-\left(u_{0}\right)_{x}^{2}}{\epsilon} d x\right. \\
\left.\quad-\frac{1}{2} \int_{0}^{1}\left[B_{1} \frac{\left(u_{0}+\epsilon l\right)^{2}-u_{0}^{2}}{\epsilon}+B_{2} \frac{\left(a-\left(u_{0}+\epsilon l\right)\right)^{2}-\left(a-u_{0}\right)^{2}}{\epsilon}\right] d x\right\} \\
=\int_{0}^{1}\left[\left(A_{1} \psi_{1}+A_{2} \psi_{2}\right)(x, T)-\left(u_{0}\right)_{x} l_{x}-B_{1} u_{0} l+B_{2}\left(a-u_{0}\right) l\right] d x \\
=\int_{0}^{1}\left[\left(p \psi_{1}+q \psi_{2}\right)(x, T)-\left(u_{0}\right)_{x} l_{x}-B_{1} u_{0} l+B_{2}\left(a-u_{0}\right) l\right] d x \\
=\int_{0}^{1}\left(p \psi_{1}(x, 0)+q \psi_{2}(x, 0)-\left(u_{0}\right)_{x} l_{x}-B_{1} u_{0} l+B_{2}\left(a-u_{0}\right) l\right) d x \\
\quad+\int_{0}^{T} \int_{0}^{1}\left[p\left(\psi_{1}\right)_{t}+\left(r_{1}\left(k_{1}-2 u\right)-k_{3} w\right) p+\alpha_{1} k_{3} w r\right] \psi_{1} d x d t \\
\quad+\int_{0}^{T} \int_{0}^{1}\left[q\left(\psi_{2}\right)_{t}+\left(r_{2}\left(k_{2}-2 v\right)-k_{2} w\right) q+\alpha_{2} k_{4} w r\right] \psi_{2} d x d t \\
+\int_{0}^{T} \int_{0}^{1}\left[r\left(\psi_{3}\right)_{t}+d_{1}\left(\psi_{3}\right)_{x} r_{x}-k_{3} u p-k_{4} v q+\left(\alpha_{1} k_{3} u+\alpha_{2} k_{4} v-\mu\right) r\right] \psi_{3} d x d t \\
=\int_{0}^{1}\left[\left(p \psi_{1}+q \psi_{2}\right)(x, 0)-\left(u_{0}\right)_{x} l_{x}-B_{1} u_{0} l+B_{2}\left(a-u_{0}\right) l\right] d x+\int_{Q}(p, q, r) \mathcal{L}\left(\begin{array}{c}
\psi_{2} \\
\psi_{3}
\end{array}\right) d x d t \\
=\int_{0}^{1}\left[l p(x, 0)-l q(x, 0)-\left(u_{0}\right)_{x} l_{x}-B_{1} u_{0} l+B_{2}\left(a-u_{0}\right) l\right] d x
\end{gathered}
$$


The above inequality can be re-written in a formal way as (after integrating by parts the $\left(u_{0}\right)_{x} l_{x}$ term) :

$$
\int_{0}^{1} l\left[p(x, 0)-q(x, 0)+\left(u_{0}\right)_{x x}-B_{1} u_{0}+B_{2}\left(a-u_{0}\right)\right] d x \leq 0
$$

On the set where $u_{0}=0$, we choose variation $l$ with support on this set and $l \geq 0$, which implies $-\left(p(x, 0)-q(x, 0)+\left(u_{0}\right)_{x x}-B_{1} u_{0}+B_{2}\left(a-u_{0}\right)\right) \geq 0$.

$$
\begin{array}{lll}
0<u_{0}<a, & l \text { arb sign, } & -\left(p(x, 0)-q(x, 0)+\left(u_{0}\right)_{x x}-B_{1} u_{0}+B_{2}\left(a-u_{0}\right)\right)=0 \\
u_{0}=a, & l \leq 0, & -\left(p(x, 0)-q(x, 0)+\left(u_{0}\right)_{x x}-B_{1} u_{0}+B_{2}\left(a-u_{0}\right)\right) \leq 0
\end{array}
$$

This can be written in the compact form as

$$
\min \left\{\max \left(-\left(p(x, 0)-q(x, 0)+\left(u_{0}\right)_{x x}-B_{1} u_{0}+B_{2}\left(a-u_{0}\right)\right), u_{0}-a\right), u_{0}-0\right\}=0 .
$$

Our optimality system (OS) is :

$$
\begin{aligned}
& \mathcal{L}_{1} u=r_{1} u\left(k_{1}-u\right)-k_{3} u w \\
& \mathcal{L}_{2} v=r_{2} v\left(k_{2}-v\right)-k_{4} v w \\
& \mathcal{L}_{3} w=\alpha_{1} k_{3} u w+\alpha_{2} k_{4} v w-\mu w \\
& \mathcal{L}_{1}^{*} p=\left(r_{1}\left(k_{1}-2 u\right)-k_{3} w\right) p+\alpha_{1} k_{3} w r \\
& \mathcal{L}_{2}^{*} q=\left(r_{2}\left(k_{2}-2 v\right)-k_{4} w\right) q+\alpha_{2} k_{4} w r \\
& \mathcal{L}_{3}^{*} r=-k_{3} u p-k_{4} v q+\left(\alpha_{1} k_{3} u+\alpha_{2} k_{4} v-\mu\right) r \\
& \min \left\{\max \left(-\left(p(x, 0)-q(x, 0)+\left(u_{0}\right)_{x x}-B_{1} u_{0}+B_{2}\left(a-u_{0}\right)\right), u_{0}-a\right), u_{0}-0\right\}=0 \\
& u(x, 0)=u_{0}(x), \quad v(x, 0)=v_{0}(x), \quad w(x, 0)=w_{0}(x) \quad \text { for } x \in \Omega \\
& w(0, t)=0=w(1, t), \quad r(0, t)=0=r(1, t) \quad \text { on }(0, T) \\
& p(x, T)=A_{1}, \quad q(x, T)=A_{2}, \quad r(x, T)=0 \quad \text { for } x \in \Omega \\
& r(0, t)=0=r(1, t) \quad \text { where } t \in[0, T] .
\end{aligned}
$$

The weak solution of the optimality system exists by Theorem 2.2 and 3.2. For small $T$, we now prove the uniqueness of weak solutions of optimality system, which gives the characterization (3.2) of the unique optimal control in terms of the solutions of the optimality system. Note that such a small $T$ restriction is common in optimal control problems involving parabolic PDEs; see the uniqueness results in optimal control of the PDE/ODE systems in $[2,6]$.

\section{Uniqueness of the Optimality System}

Theorem 4.1 For $T$ sufficiently small and $B_{1}+B_{2}$ sufficiently large, weak solutions of the optimality system are unique. 


\section{Proof :}

Suppose $u, v, w, p, q, r, u_{0}$ and $\bar{u}, \bar{v}, \bar{w}, \bar{p}, \bar{q}, \bar{u}_{0}$ are two solutions of the optimal system (3.2). We change the variables for $\lambda>0$ to be chosen such that

$$
\begin{array}{ll}
u=e^{\lambda t} u_{1} & \bar{u}=e^{\lambda t} u_{2} \\
v=e^{\lambda t} v_{1} & \bar{v}=e^{\lambda t} v_{2} \\
w=e^{\lambda t} w_{1} & \bar{w}=e^{\lambda t} w_{2} \\
p=e^{-\lambda t} p_{1} & \bar{p}=e^{-\lambda t} p_{2} \\
v=e^{-\lambda t} q_{1} & \bar{q}=e^{-\lambda t} q_{2} \\
r=e^{-\lambda t} r_{1} & \bar{r}=e^{-\lambda t} r_{2} .
\end{array}
$$

The variational inequality for $u_{0}$ becomes

$$
\int_{\Omega}\left[\left(u_{0}\right)_{x}\left(v_{0}-u_{0}\right)_{x}+\left\{e^{-\lambda t}\left(q_{1}-p_{1}\right)(x, 0)+B_{1} u_{0}-B_{2}\left(a-u_{0}\right)\right\}\left(v_{0}-u_{0}\right)\right] d x \geq 0 .
$$

Substituting $v_{0}=\bar{u}_{0}$ in the $u_{0}$ variational inequality, and adding the resulting inequalities, we obtain

$$
\begin{gathered}
\int_{\Omega}\left(u_{0}-\bar{u}_{0}\right)_{x}^{2}+\left(B_{1}+B_{2}\right)\left(u_{0}-\bar{u}_{0}\right)^{2} \\
\leq \int_{\Omega} e^{-\lambda t}\left(u_{0}-\bar{u}_{0}\right)\left(\left(q_{1}-q_{2}\right)-\left(p_{1}-p_{2}\right)\right)(x, 0) d x .
\end{gathered}
$$

Using the weak form of the state and adjoint systems and the above inequality, gives

$$
\begin{gathered}
\int_{\Omega}\left[\left(p_{1}-p_{2}\right)^{2}+\left(q_{1}-q_{2}\right)^{2}+\left(r_{1}-r_{2}\right)^{2}\right](x, 0) d x \\
+\int_{\Omega}\left[\left(u_{1}-u_{2}\right)^{2}+\left(v_{1}-v_{2}\right)^{2}+\left(w_{1}-w_{2}\right)^{2}\right](x, T) d x \\
+\lambda \int_{Q}\left[\left(u_{1}-u_{2}\right)^{2}+\left(v_{1}-v_{2}\right)^{2}+\left(w_{1}-w_{2}\right)^{2}+\left(p_{1}-p_{2}\right)^{2}+\left(q_{1}-q_{2}\right)^{2}+\left(r_{1}-r_{2}\right)^{2}\right] d x d t \\
+\int_{\Omega}\left[\left(\left(u_{0}-\bar{u}_{0}\right)_{x}\right)^{2}+\left(B_{1}+B_{2}\right)\left(u_{0}-\bar{u}_{0}\right)^{2}\right] d x+\int_{Q} d_{1}\left(\left|\left(w_{1}-w_{2}\right)_{x}\right|^{2}+\left|\left(r_{1}-r_{2}\right)_{x}\right|^{2}\right) d x d t \\
\leq\left(C_{1}+C_{2} e^{2 \lambda T}\right)\left[\int _ { Q } \left\{\left(u_{1}-u_{2}\right)^{2}+\left(v_{1}-v_{2}\right)^{2}+\left(w_{1}-w_{2}\right)^{2}\right.\right. \\
\left.\left.+\left(p_{1}-p_{2}\right)^{2}+\left(q_{1}-q_{2}\right)^{2}+\left(r_{1}-r_{2}\right)^{2}\right\} d x d t\right] \\
+\int_{\Omega} \frac{1}{2}\left[\left(p_{1}-p_{2}\right)^{2}+\left(q_{1}-q_{2}\right)^{2}\right](x, 0) d x+\frac{1}{2} \int_{\Omega}\left(u_{0}-\bar{u}_{0}\right)^{2} d x+\frac{1}{2} \int_{\Omega}\left(u_{0}-\bar{u}_{0}\right)^{2} d x,
\end{gathered}
$$

where the last term comes from the initial conditions on $u, v, \bar{u}, \bar{v}$. If we take $\lambda$ large and then $T$ small, we have

$$
\lambda-C_{1}-C_{2} e^{2 \lambda T}>0 .
$$

If we also assume $B_{1}+B_{2}>\frac{5}{2}$, then we obtain the uniqueness. 


\section{Numerical Realization}

We perform the numerical realization of the problem using the optimality system (3.2) that we recall thereafter :

$$
\begin{aligned}
& \left\{\begin{array}{rlrl}
\frac{d u}{d t} & =r_{1} u\left(k_{1}-u\right)-k_{3} u w, & u(0)=u_{o} & \\
\frac{d v}{d t}=r_{2} v\left(k_{2}-v\right)-k_{4} u w, & v(0)=a-u_{o} & \\
\frac{\partial w}{\partial t}=d_{1} \frac{\partial^{2} w}{\partial x^{2}}+\left(\alpha_{1} k_{3} u+\alpha_{2} k_{4} v-\mu\right) w, & w_{\mid x=0, x=1}=0, & w(0)=w_{o}
\end{array}\right. \\
& \left\{\begin{array}{rlrl}
-\frac{d p}{d t} & =\left(r_{1}\left(k_{1}-2 u\right)-k_{3} w\right) p+\alpha_{1} k_{3} w r, & & p(T)=A_{1} \\
-\frac{d q}{d t}=\left(r_{2}\left(k_{2}-2 v\right)-k_{4} w\right) q+\alpha_{2} k_{4} w r, & & q(T)=A_{2} \\
-\frac{\partial r}{\partial t}=d_{1} \frac{\partial^{2} r}{\partial x^{2}}+\left(\alpha_{1} k_{3} u+\alpha_{2} k_{4} v-\mu\right) r-k_{3} u p-k_{4} v q & & r_{\mid x=0, x=1}=0
\end{array}\right. \\
& \min _{0 \leq u_{o} \leq a} \frac{1}{2} \int_{0}^{1}\left[\frac{d u_{o}}{d x}\right]^{2}+\left(B_{1}+B_{2}\right) u_{o}^{2}-2\left(a B_{2}+p(0)-q(0)\right) u_{o} d x
\end{aligned}
$$

In the sequel, we set $B=B_{1}+B_{2}$ and $f=\left(B_{2}+p(0)-q(0)\right)$.

These equations are coupled and we are going to solve this system via a relaxation method that can be roughly described a follows :

\section{Relaxation forward-backward method}

\section{Initialization step Choose $u_{o}$}

2. Iteration $n: u_{n}$ is known.

(a) Solve the forward system $(5.1)$ : we get $\left(u_{n}, v_{n}, w_{n}\right)$.

(b) Solve the backward system (5.2) with $\left(u_{n}, v_{n}, w_{n}\right)$ : we get $\left(p_{n}, q_{n}, r_{n}\right)$.

(c) Solve the Variational Inequality (5.3) with $f_{n}=B_{2}+p_{n}(0)-q_{n}(0)$ : we get $u_{n+1}$.

3. Check a stopping criterion and set $n=n+1$ if necessary.

The discretization of the system is done via finite difference methods with respect to the time variable and the space variable. The implicit Euler scheme is used to solve the forward system ODE's and the space-discretized part of PDE's and the Crank-Nicholson scheme is used for the backward (linear) system. This choice has been made using many numerical tests : though the Crank-Nicholson scheme is inconditionnally stable we could not avoid scattering for some examples that were particularly ill-conditionned. The state system (5.1) is a non-linear system, we use Newton's method to solve it. The initial point 
is chosen as the previous iterate so that the convergence is quite fast. Note that the ratio between the time discretization step and the space discretization step has to be small enough (CFL condition) to avoid numerical unstability.

To solve the Variational Inequality (5.3), we discretize the energy functional with the trapezoidal integration rule (for example) and use the classical projected gradient method to solve it. Indeed the functional is quadratic and constraints are bound constraints. The discretized problem turns out to be

$$
\min \frac{1}{2} X^{\prime} \mathcal{M} X-F^{\prime} X, 0 \leq X_{i} \leq a, i=1, \cdots, N+1
$$

where $X=\left(u_{o}\left(x_{i}\right)\right)_{i=1, N}$ is the discretized control function, $X^{\prime}$ denotes the transpose of $X, F$ is the (space) discretized vector for $f=B_{2}+p(0)-q(0)$ and $\mathcal{M}=\mathcal{A}+B I d$. Also $\mathcal{A}$ is the discretized $1 \mathrm{D}$ - Laplacian matrix :

$$
\mathcal{A}=\frac{1}{(\Delta x)^{2}}\left[\begin{array}{ccccc}
2 & -1 & 0 & \cdots & \cdots \\
-1 & 2 & -1 & 0 & \vdots \\
0 & -1 & 2 & -1 & \vdots \\
\vdots & \ddots & \ddots & \ddots & \vdots \\
\vdots & 0 & -1 & 2 & -1 \\
\cdots & \cdots & \cdots & -1 & 2
\end{array}\right]
$$

We have performed numerical tests with the following parameters :

\begin{tabular}{|c|c||c||c|c||c|c|c|c|c||c||c|c|c|c||c|}
\hline$r_{1}$ & $r_{2}$ & $d_{1}$ & $k_{1}$ & $k_{2}$ & $\alpha_{1}$ & $\alpha_{2}$ & $k_{3}$ & $k_{4}$ & $\mu$ & $\mathrm{a}$ & $A_{1}$ & $A_{2}$ & $B_{1}$ & $B_{2}$ & $\mathrm{~T}$ \\
\hline 0.5 & 0.5 & 1 & 1 & 1 & 0.25 & 0.25 & 0.4 & 0.4 & 0.2 & 1 & 2 & 1 & 1 & 1 & 1 \\
\hline
\end{tabular}

We have set $w_{o}(x)=10 x(1-x), N=200, K=10$ and the initial guess for $u_{o}$ is equal to 0.5 ; the tolerance has been set to $10^{-4}$ and the parameter of projected gradient method is $\rho=0.5$. We report hereafter the results.

\begin{tabular}{|c|c|c|}
\hline Global iteration $n$ & \# of projected gradient iterations & $\left\|u_{o}^{n}-u_{o}^{n-1}\right\|$ \\
\hline 1 & $1.852 \mathrm{e}+04$ & $4.960937 \mathrm{e}+00$ \\
\hline 2 & 12 & $1.200432 \mathrm{e}-03$ \\
\hline 3 & 1 & $9.998819 \mathrm{e}-05$ \\
\hline
\end{tabular}

So the global iterations number is 3 and the value of the cost functional is $J^{*}=1.591363$. 


\section{Références}

[1] Ames, W. F., Numerical Methods for Partial Differential Equations, Barnes and Noble, 1969 .

[2] S. Chawla and S. Lenhart, Application of Optimal Control Theory to in situ Bioremediation, IMA Preprint \# 1419, Computational and Applied Math Journal 114(2000), $81-102$.

[3] M. Chipot,Variational Inequalities and Flow in Porous Media, Springer-Verlag, New York, 1984.

[4] Evans, L.C., Partial Differential Equations, American Mathematical Society, Providence, 1998.

[5] Federer,W. T., Statistical Design and Analysis for Intercropping Experiments Volume I : Two Crops, Springer-Verlag, New York, 1993.

[6] N. Handagama and S. Lenhart, Optimal Control of a PDE/ ODE System Modeling a GasPhase Bioreactor, referred Proceedings of the Conference on Mathematical Models in Medical and Health Sciences, Vanderbilt University, 1998, 197 - 212.

[7] D. Kinderlehrer and G. Stampacchia, An Introduction to Variational Inequalities and their Applications, Academic Press, New York, 1980.

[8] Liebman, M. and E. Dyck, Crop Rotation and Intercropping Strategies for Weed Management, Ecological Applications 3 (1993), 92-122.

[9] Plant, R. E. and M. Mangel, Modeling and Simulation in Agricultural Pest Management, SIAM Review 29 (1987), 235-261.

[10] Simon, J., Compact sets in the space $L^{2}(0, T ; B)$, Annali di Matematica Pura et Applicata CXLVI (1987), 65-96. 